\title{
Epidemiología del hipotiroidismo congénito en México
}

Marcela Vela-A mieva, MC, ${ }^{(1)}$ Salvador Gamboa-Cardiel, Biól, ${ }^{(1)}$ Martha E Pérez-Andrade, Q FB, ${ }^{(1)}$ Joel 0 rtiz-Cortés, Q FB, ${ }^{(1)}$ Claudia R González-Contreras, Q C, ${ }^{(1)}$ Venancio 0 rtega-Velázquez, Q FB., ${ }^{(1)}$

\begin{abstract}
Vela-Amieva M, Gamboa-Cardiel S, Pérez-Andrade ME, Ortiz-Cortés J, González-Contreras CR, Ortega-Velázquez V. Epidemiología del hipotiroidismo congénito en México. Salud Publica Mex 2004;46:141-148.

El texto completo en inglés de este artículo está disponible en: http://www.insp.mx/salud/index.html
\end{abstract}

\section{Resumen}

Objetivo. Describir las características epidemiológicas del hipotiroidismo congénito en recién nacidos en unidades médicas de la Secretaría de Salud de México. Material y métodos. Estudio transversal en el cual se cuantificó tirotropina en 1379717 muestras de sangre de recién nacidos (de cordón umbilical o de talón), recolectadas en papel filtro, de enero de 2001 a diciembre de 2002, en unidades médicas de la Secretaría de Salud. Las muestras con tirotropina $\geq 15 \mu \mathrm{Ul} / \mathrm{ml}$ para sangre de cordón, $\mathrm{y} \geq 10 \mu \mathrm{Ul} / \mathrm{ml}$ para talón, se consideraron sospechosas, y se notificaron para realizar el perfil tiroideo y gammagrafía. C uando los resultados fueron positivos, el caso se registró como hipotiroidismo congénito confirmado, y se anotaron los datos clínicos y demográficos en formatos específicos. Se utilizó estadística descriptiva estándar. Resultados. Se encontró una prevalencia de $4.12 \times 10000$ recién nacidos, con predominancia del sexo femenino (66.84\%). Se observaron variaciones estatales en la prevalencia, máxima en Q uintana Roo ( 8.13 x 10000 recién nacidos) y mínima en Sinaloa $(0.62 \times 10000)$. Se encontraron $57.46 \%$ tiroides ectópicas, $35.91 \%$ agenesias tiroideas y $6.63 \%$ defectos de la función de las hormonas tiroideas. Los principales datos clínicos fueron hernia umbilical (43.73\%) e ictericia (41.58\%).A 151 (17.12\%) sospechosos no se les realizó estudio confirmatorio debido a datos incompletos o falsos en la ficha de identificación, fallecimiento del recién nacido, renuencia de los padres y cambio de domicilio. Conclusiones. El hipoti-

\author{
Vela-Amieva M, Gamboa-Cardiel S, Pérez-Andrade ME, \\ Ortiz-Cortés J, González-Contreras CR, Ortega-Velázquez V. \\ Epidemiology of congenital hypothyroidism in Mexico. \\ Salud Publica Mex 2004;46:141-148. \\ The English version of this paper \\ is available at: http://www.insp.mx/salud/index.html
}

\begin{abstract}
A bstract
Objective. To describe the epidemiological characteristics of congenital hypothyroidism (CH) in newborns (NB) detected by the Ministry of Health of Mexico (SSA). Material and Methods. A cross-sectional study was conducted. Thyroid stimulating hormone (TSH) was quantified in 1379717 blood samples of N B (from umbilical cord or heel prick), collected in G uthrie cards, between January 2001 and December 2002, in medical units of the SSA.The samples with TSH $\geq 15 \mathrm{mUl} / \mathrm{ml}$ for cord blood and $\geq 10 \mathrm{mUI} / \mathrm{ml}$ for heel blood were considered suspicious and were submitted for confirmation by thyroid hormone profile and/or scintigraphy. When the results were positive, the case was registered as confirmed $\mathrm{CH}$ and the clinical and demographic data were recorded in specific formats. Statistical analysis was performed using descriptive statistics. Results. The prevalence of $\mathrm{CH}$ was $4.12 \times 10,000 \mathrm{~N} \mathrm{~B}$, with female predominance $(66.84 \%)$. Regional variations in the prevalence were observed, the highest in Q uintana Roo $(8.13 \times 10000 \mathrm{~N} \mathrm{~B})$ and the lowest in Sinaloa $(0.62 \times 10,000 \mathrm{~N}$ B). Abnormalities included $57.46 \%$ of ectopic glands, $35.91 \%$ of athyrosis, and $6.63 \%$ abnormal thyroid function conditions. The main clinical features were umbilical hernia $(43.73 \%)$ and jaundice (41.58\%). A total of 151 (17.12\%) suspicio us N B were not confirmed because of incomplete or inaccurate identification data, death of the N B, parent refusal, and change of address. Conclusions CH was present in 1:2 $426 \mathrm{~N} \mathrm{~B}$, with a female predominance (2:1); an ectopic thyroid was the
\end{abstract}

(1) Unidad de Genética de la Nutrición, Instituto N acional de Pediatría, Centro N acional de Equidad de Género y Salud Reproductiva. Secretaría de Salud, México, DF, México.

Fecha de recibido: 10 de junio de 2003 - Fecha de aprobado: 1 de octubre de 2003

Solicitud de sobretiros: D ra. MarcelaVela A mieva. Unidad de Genética de la N utrición. A venida Insurgentes Sur 3700 C, 3er piso, colonia Insurgentes C uicuilco, C oyoacán, 04530 México, D F, México.

Correo electrónico: amieva@ servidor.unam.mx 
roidismo se presentó en 1:2 426 recién nacidos, con predominio femenino (2:1), siendo la ectopia tiroidea su forma más común, y la hernia umbilical y la ictericia sus datos clínicos prominentes. El texto completo en inglés de este artículo está disponible en:http://www.insp.mx/salud/index.html

Palabras clave: hipotiroidismo congénito; tamiz neonatal; defectos al nacimiento; retraso mental; prevención; México most common finding, and its prominent clinical features were umbilical hernia and jaundice. The English version of this paper is available at:http://w ww.insp.mx/salud/index.html

Key words: congenital hypothyroidism; neonatal screening; hypothyroidism frequency; Mexico
E 1 hipotiroidismo congénito (HTC) es un defecto al nacimiento que constituye una urgencia pediátrica que, cuando no recibe tratamiento oportuno, tiene consecuencias graves entre las que destacan el retraso mental irreversible. ${ }^{1}$ La historia natural del HTC ha cambiado dramáticamente en los últimos años gracias a los programas de tamiz neonatal (TN), que consisten en detectar la enfermedad en todos los recién nacidos $(\mathrm{RN})$ aparentemente sanos. ${ }^{2,3}$

En nuestro país el TN se inició formalmente en 1988 con la expedición de la Norma Técnica 321, ${ }^{4}$ y actualmente su realización es una acción obligatoria para todos los centros que brindan atención materno infantil, según lo establece la Norma Oficial Mexicana007-SSA2-1993. ${ }^{5}$ Los resultados del programa de TN de la Secretaría de Salud de México (SSA) han sido publicados con anterioridad. ${ }^{6-8}$

Mediante el TN se sabe que la prevalencia mundial de HTC es de dos a tres casos por cada 10000 (1:2 000 a 1:3 000) RN; ;, 10 sin embargo, se han descrito variaciones en la frecuencia tanto geográficas como poblacionales. Por ejemplo, Toublanc ${ }^{9}$ y otros autores sostienen que en Estados Unidos de América, en la población de origen "hispano", se llegan a presentar hasta 5.28 casos por cada $10000 \mathrm{RN}(1: 1894) .{ }^{11-15}$

La explicación de estas diferencias en la frecuencia del HTC no se conocen con precisión, sin embargo, parecen más relacionadas con los trastornos por deficiencia de yodo que con las características étnicas poblacionales. ${ }^{10,16-18}$

Las principales causas que producen HTC son: a) migración incompleta o aberrante del esbozo tiroideo, lo que ocasiona una glándula ectópica sin lóbulos laterales, esto también se conoce como nódulo tiroideo; b) diferenciación o crecimiento tiroideo defectuoso, lo cual resulta en una agenesia tiroidea o atiriosis, y c) defectos en la biosíntesis de las hormonas tiroideas, o dishormonogénesis con o sin bocio. Las dos primeras entidades se agrupan bajo el nombre de disgenesias tiroideas, las cuales son esporádicas y tienen un predominio en el sexo femenino. ${ }^{19}$
Los mecanismos moleculares involucrados con la diferenciación celular de la tiroides no se conocen con exactitud, no obstante se han descrito algunas mutaciones en genes involucrados con el desarrollo y crecimiento de dicha glándula como TTF1, TTF2, PAX8 y TSHR, entre otros. $20-26$

La predominancia femenina es una característica particularmente interesante de la epidemiología del HTC primario, sin embargo, no se sabe si las mujeres son más susceptibles de desarrollar HTC o si los fetos femeninos con HTC tienen mayor sobrevivencia uterina comparada con los masculinos. ${ }^{15}$

El objetivo del presente trabajo es describir algunas de las características epidemiológicas del HTC en los RN que se atendieron en las unidades médicas de la SSA, durante el periodo comprendido entre enero de 2001 a diciembre de 2002.

\section{Material y métodos}

Se trata de un estudio transversal en el que se incluyeron 1379717 muestras de sangre de RN mexicanos, que se recolectaron de enero de 2001 a diciembre de 2002 en unidades médicas pertenecientes a la SSA, que atienden partos y RN. Las muestras se obtuvieron mediante punción del talón entre las 48 horas y los 45 días de vida, o bien mediante punción del cordón umbilical, en los primeros 15 minutos de vida. La sangre se depositó en papel filtro (tarjeta de Guthrie) de acuerdo con las técnicas internacionalmente aceptadas; $3,27,28$ posteriormente fueron enviadas por mensajería para su evaluación y procesamiento bioquímico, que consistió en la cuantificación de hormona estimulante de la tiroides (TSH) mediante el método de ELISA, usando estuches comerciales. Se utilizaron controles de calidad externos provistos por el Centro de Control de Enfermedades (CDC) de Atlanta ${ }^{29}$ y el Programa de Evaluación Externa de Calidad (PEEC) de Argentina.

Los valores de TSH iguales o mayores de $10 \mu \mathrm{UI} / \mathrm{ml}$ para muestras provenientes de sangre de talón, y de $15 \mu \mathrm{UI} / \mathrm{ml}$ para sangre de cordón umbilical de los 
RN fueron considerados casos sospechosos. Todas las muestras elevadas se procesaron por duplicado, $\mathrm{y}$ aquellas con valores consistentemente altos se notificaron para su localización y para la realización de exámenes confirmatorios (perfil tiroideo o gammagrafía). Cuando los resultados fueron positivos, el RN se registró como caso confirmado de HTC; cuando dichos resultados fueron negativos, el $\mathrm{RN}$ se consideró como falso positivo. Se definió como caso no confirmado atodo aquel $\mathrm{RN}$ sospechoso que, a pesar de haber sido localizado, no se le pudo realizar la prueba confirmatoria. Por último, se consideró caso no localizado a todos aquellos sospechosos que no pudieron ser ubicados.

Los datos demográficos fueron consignados en formatos específicos (ficha de identificación, notificación de sospechoso, resultado de la notificación con perfil tiroideo y estudio gammagráfico). $\mathrm{Al}$ momento del estudio confirmatorio de cada caso se realizó una revisión clínica de los pacientes y se llenó un formato para asentar las siguientes características clínicas: facies tosca, fontanela amplia, ictericia, edema, macroglosia, llanto ronco, somnolencia, hipotonía, piel seca, hipotermia, hipoactividad, estreñimiento, hernia umbilical y lentitud en la ingesta de los alimentos. La prevalencia de HTC se calculó como el número de casos confirmados durante el periodo comprendido de enero de 2001 a diciembre de 2002 sobre el número total de $\mathrm{RN}$ tamizados adecuadamente en dicho periodo multiplicado por $10000 .^{30}$

Tasa de prevalencia $=\frac{\text { Total de casos confirmados }}{\text { Total de tamizados adecuados }} \times 10000$

La tasa de falla (estimación del número de casos perdidos) se obtuvo mediante la fórmula:

Tasa de falla $=\frac{\text { No confirmados }+ \text { no localizados }}{\text { Total de tamizados adecuados }} \times 10000$

En el caso de los sospechosos que no se pudieron confirmar debido a fallecimiento se procedió a solicitar el certificado de defunción correspondiente.

Para el análisis estadístico se utilizó estadística descriptiva estándar, las variables categóricas se reportan en proporciones; para el análisis de regresión logística se utilizó el programa MedCalc.

\section{Resultados}

De $1379717 \mathrm{RN}$ a los que se les realizó la toma de sangre en papel filtro, 67\% (924 410) de las muestras fueron de cordón umbilical y 33\% (455 307) del talón.
Se obtuvieron 1354102 (98.14\%) muestras adecuadas para el procesamiento bioquímico, y 25615 (1.85\%) no cumplieron con los criterios de calidad para ser analizadas debido a que presentaron alguno de los siguientes problemas: papel filtro sobresaturado de sangre, gotas de sangre insuficiente, y falla en la elución (extracción y liberación de la sangre del medio sólido, en este caso el papel de algodón, que la ha absorbido).

Encontramos 882 neonatos sospechosos con valores elevados de TSH, y las pruebas confirmatorias se pudieron realizar únicamente en 731 (82.88\%) de ellos. Los 151 (17.12\%) niños restantes no se pudieron confirmar debido a diversos motivos, entre los que destacan 64 casos por datos incompletos o falsos en la ficha de identificación, 42 por fallecimiento del neonato sospechoso, 30 por renuencia de los padres a la realización del estudio y 15 por cambio de domicilio. Las causas de fallecimiento de los 42 sospechosos se muestran en el cuadro I, en donde también se señala que 26 fueron femeninos y 16 masculinos.

La edad promedio de las madres de los casos confirmados de HTC fue de 25.38 años (mínimo de 14 y máximo de 49). La duración promedio de la gestación fue de 39.35 semanas (mínimo 29 y máximo 43), el peso

\section{Cuadro I \\ Causas de fallecimiento de los recién nacidos SOSPECHOSOS DE HIPOTIROIDISMO CONGÉNITO. MÉxıco, 2001-2002}

\begin{tabular}{lccc} 
Causa de muerte & Femenino & Masculino & Total \\
A sfixia neonatal severa & 1 & 4 & 5 \\
\hline Falla orgánica múltiple & 1 & 3 & 4 \\
\hline Hemorragia intracraneal & 1 & 1 & 2 \\
\hline Hemorragia pulmonar & 2 & & 2 \\
\hline Choque séptico severo & 1 & 1 & 2 \\
\hline Broncoaspiración & 2 & & 2 \\
\hline Paro respiratorio & 1 & & 1 \\
\hline Depresión neonatal severa & & 1 & 1 \\
\hline Desnutrición & 1 & & 1 \\
\hline Falla cardiaca & 1 & & 1 \\
\hline Gastroenteritis & & 1 & 1 \\
\hline $\begin{array}{l}\text { Miocardiopatía hipóxica } \\
\text { N eumonía basal }\end{array}$ & 1 & & 1 \\
\hline $\begin{array}{l}\text { Insuficiencia respiratoria } \\
\text { Se desconocen las causas* }\end{array}$ & 1 & & 1 \\
\hline Total & 12 & 5 & 17 \\
$*$ No se pudo obtener el certificado de defunción & & \\
& & & \\
\hline
\end{tabular}


promedio de los RN fue de 3298 g (mínimo de 860 y máximo de 5 900). De los casos confirmados de HTC, $373(66.85 \%)$ fueron del sexo femenino y $185(33.15 \%)$ del masculino. Se obtuvo información completa de los datos clínicos, al momento de la confirmación diagnóstica, únicamente en 399 casos. La característica clínica que se encontró con mayor frecuencia fue la hernia umbilical, misma que estuvo presente en $43.73 \%$ de los afectados, seguida de ictericia en $41.58 \%$; piel seca, $36.92 \%$; estreñimiento, $36.74 \%$; facies tosca, $36.20 \%$; llanto ronco, $34.05 \%$; fontanela posterior amplia, $33.87 \%$; edema palpebral, $32.08 \%$; y macroglosia, $29.21 \%$. La frecuencia de otros datos clínicos se muestra en el cuadro II.

Se pudo conocer la etiología del HTC exclusivamente en 181 casos en los cuales se elaboró el estudio gammagráfico, de lo que se encontró que $57.46 \%$ de los pacientes correspondieron a glándula ectópica; $35.91 \%$, agenesia tiroidea; $3.87 \%$; bocio, y $2.76 \%$, dishormonogénesis. La predominancia del sexo femenino tanto en la ectopia como en la agenesia tiroidea es estadísticamente significativa (cuadro III).

En el cuadro IV se muestran, en forma desglosada por entidad federativa, el total de $\mathrm{RN}$ atendidos por la SSA (1 361 850), así como la cobertura (101.31\%), los RN tamizados (1 379 717), el número de casos de HTC (558) y sus respectivas tasas de prevalencia $(4.12 \times 10000)$ y de falla $(1.09 \times 10000)$. Por último, en el cuadro $\mathrm{V}$ se observan los tiempos promedio que se obtuvieron para el inicio de tratamiento a los recién nacidos confirmados, que fueron 26.25 días mayor al ideal (30 días).

Se observó variabilidad en la prevalencia de la enfermedad en los distintos estados de la República, que llegó a una tasa máxima de 8.13 x 10000 RN en Quintana Roo, seguida de 7.78 en San Luis Potosí y 7.53 en Baja California Sur; por el contrario, el estado que tuvo la tasa más baja fue Sinaloa $(0.62$ X 10 000). En el caso de Yucatán no se pudo calcular esta tasa ya que no se confirmó ningún caso, a pesar de tener 16 sospechosos; por lo tanto, presentó el mayor índice de falla (6.10 X 10000$)$ de toda la muestra analizada.

\section{Discusión}

Desde que iniciaron los programas de TN para HTC en el mundo, se estima que se han analizado más de 150 millones de $\mathrm{RN}$ y se han descubierto alrededor de 42000 afectados (1:3 571). ${ }^{2}$ En México, desde 1989 a la fecha se han tamizado en la SSA 4052782 niños con la detección de 1576 casos (1:2,572); en los últimos años la búsqueda de esta enfermedad ha mejorado tanto bioquímica como operativamente, lo cual ha repercutido

\section{Cuadro II \\ Datos clínicos al momento del diagnóstico DE hiPOTIROIDISMO CONGÉNITO. MÉxico, 2001-2002}

\begin{tabular}{lc} 
Datos clínicos & Frecuencia \\
Hernia umbilical & $244(43.73 \%)$ \\
\hline Ictericia & $232(41.58 \%)$ \\
\hline Piel seca & $206(36.92 \%)$ \\
\hline Estreñimiento & $205(36.74 \%)$ \\
\hline Facies tosca & $202(36.20 \%)$ \\
\hline Llanto ronco & $190(34.05 \%)$ \\
\hline Fontanela posamplia & $189(33.87 \%)$ \\
\hline Edema & $179(32.08 \%)$ \\
\hline Macroglosia & $163(29.21 \%)$ \\
\hline Somnolencia & $148(26.52 \%)$ \\
\hline Hipoactividad & $134(24.01 \%)$ \\
\hline Hipotonía & $131(23.48 \%)$ \\
\hline Lentitud de ingesta & $109(19.53 \%)$ \\
\hline Hipotermia & $72(12.90 \%)$ \\
\hline Sin registro de datos clínicos & $159(28.49 \%)$ \\
$n=558$ &
\end{tabular}

\section{Cuadro III \\ Etiología de los Casos comprobados DEL HIPOTIROIDISMO CONGÉNITO, OBTENIDA MEDIANTE La gammagrafía. México, 2001-2002}

\begin{tabular}{|c|c|c|c|c|c|c|}
\hline Etiología & & Total & Femenino & & sculino & p \\
\hline Ectopia & 104 & $57.46 \%$ & $7572.11 \%$ & 29 & $27.88 \%$ & $<0.0001$ \\
\hline Agenesia & 65 & $35.91 \%$ & $4670.76 \%$ & 19 & $29.23 \%$ & $<0.0001$ \\
\hline \multicolumn{7}{|c|}{ Bocio y dishormonogénesis } \\
\hline (gammagrama normal) & 12 & $6.63 \%$ & $758.33 \%$ & 5 & $41.66 \%$ & 0.6830 \\
\hline
\end{tabular}

en el hallazgo de un mayor número de enfermos y, por ende, de tasas de prevalencia más altas. Sin embargo, para hablar con certeza de la verdadera frecuencia de la enfermedad tendríamos que contar con una cobertura total, es decir, practicarles la prueba a todos los $\mathrm{RN}$ sin excepción y con cero fallas de localización de casos sospechosos, hecho que aún no se ha podido alcanzar cabalmente. A pesar de esto, el contar con una muestra como la del presente estudio en la cual la proporción de casos sospechosos en los que se logró la prueba confirmatoria fue de $82.88 \%$, y que tuvo una tasa de falla global (estimación de casos perdidos) de 


\section{Cuadro IV}

\section{Cantidad de niños estudiados en cada uno de los estados de la República Mexicana (2001-2002)}

\begin{tabular}{|c|c|c|c|c|c|c|c|c|c|c|c|c|}
\hline Estados & $\begin{array}{l}\text { RN vivos } \\
\text { de la SSA* }\end{array}$ & $\begin{array}{c}\text { Cobertura } \\
\%^{\ddagger}\end{array}$ & Tamizados & Adecuados & Inadecuados & $\begin{array}{c}\text { Total de } \\
\text { sospechosos }\end{array}$ & $\begin{array}{l}\text { Falsos } \\
\text { positivos }\end{array}$ & $\begin{array}{l}\text { No } \\
\text { confirm. }\end{array}$ & $\begin{array}{l}\text { No } \\
\text { Local. }\end{array}$ & HTC & $\begin{array}{l}\text { Tasa de prevalencia } \\
\quad \times 10000\end{array}$ & $\begin{array}{c}\text { Tasa de falla } \\
\times 10000\end{array}$ \\
\hline A guascalientes & 21053 & 86.86 & 18286 & 17989 & 297 & 15 & 7 & 2 & 0 & 6 & 3.33 & 1.09 \\
\hline Baja California & 28933 & 139.33 & 40311 & 38860 & 1451 & 30 & 14 & 4 & 2 & 10 & 2.57 & 1.48 \\
\hline Baja California Sur & 8596 & 109.59 & 9420 & 9296 & 124 & 12 & 5 & 0 & 0 & 7 & 7.53 & 0 \\
\hline Campeche & 13482 & 102.26 & 13787 & 13424 & 363 & 19 & 6 & 1 & 3 & 9 & 6.70 & 2.90 \\
\hline Coahuila & 21018 & 97.05 & 20398 & 20197 & 201 & 15 & 9 & 0 & 0 & 6 & 2.97 & 0 \\
\hline Colima & 10531 & 110.62 & 11649 & 11038 & 611 & 12 & 6 & 0 & 0 & 6 & 5.43 & 0 \\
\hline Chiapas & 58104 & 70.98 & 41245 & 38333 & 2912 & 40 & 10 & 6 & 14 & 10 & 2.60 & 4.84 \\
\hline Chihuahua & 25551 & 64.64 & 16517 & 15641 & 876 & 17 & 5 & 6 & 1 & 5 & 3.19 & 4.23 \\
\hline Distrito Federal & 128038 & 121.84 & 156001 & 154412 & 1589 & 103 & 3 & 5 & 3 & 92 & 5.95 & 0.51 \\
\hline Durango & 21054 & 106.47 & 22417 & 21488 & 929 & 15 & 9 & 1 & 0 & 5 & 2.32 & 0.44 \\
\hline Guanajuato & 78706 & 96.87 & 76242 & 74904 & 1338 & 45 & 13 & 6 & 5 & 21 & 2.80 & 1.44 \\
\hline Guerrero & 58777 & 116.56 & 68510 & 66855 & 1655 & 32 & 7 & 3 & 0 & 22 & 3.29 & 0.43 \\
\hline Hidalgo & 34810 & 128.40 & 44696 & 44449 & 247 & 30 & 1 & 1 & 0 & 28 & 6.29 & 0.22 \\
\hline Jalisco & 71980 & 80.65 & 58049 & 56913 & 1136 & 25 & 5 & 2 & 3 & 15 & 2.63 & 0.86 \\
\hline México & 129482 & 124.34 & 160993 & 159690 & 1303 & 86 & 5 & 6 & 7 & 68 & 4.25 & 0.80 \\
\hline Michoacán & 55128 & 81.90 & 45152 & 45152 & 0 & 23 & 0 & 0 & 0 & 23 & 5.09 & 0 \\
\hline Morelos & 27306 & 140.03 & 38236 & 37062 & 1174 & 13 & 0 & 0 & 0 & 13 & 3.50 & 0 \\
\hline $\mathrm{N}$ ayarit & 15440 & 121.00 & 18682 & 18429 & 253 & 16 & 5 & 2 & 0 & 9 & 4.88 & 1.07 \\
\hline N uevo León & 37538 & 106.37 & 39930 & 39930 & 0 & 17 & 0 & 0 & 0 & 17 & 4.25 & 0 \\
\hline 0 axaca & 41675 & 87.27 & 36370 & 36313 & 57 & 43 & 9 & 6 & 4 & 24 & 6.60 & 2.74 \\
\hline Puebla & 55178 & 82.70 & 45631 & 45141 & 490 & 35 & 7 & 3 & 1 & 24 & 5.31 & 0.87 \\
\hline Q uerétaro & 28519 & 117.31 & 33456 & 32823 & 633 & 35 & 11 & 10 & 3 & 11 & 3.35 & 3.88 \\
\hline Q uintana Roo & 21786 & 70.21 & 15297 & 14749 & 548 & 22 & 5 & 2 & 3 & 12 & 8.13 & 3.26 \\
\hline San Luis Potosí & 35923 & 113.18 & 40659 & 39830 & 829 & 46 & 12 & 2 & 1 & 31 & 7.78 & 0.73 \\
\hline Sinaloa & 23927 & 136.80 & 32732 & 31798 & 934 & 5 & 1 & 1 & 1 & 2 & 0.62 & 0.61 \\
\hline Sonora & 37894 & 79.49 & 30122 & 29537 & 585 & 21 & 0 & 13 & 1 & 7 & 2.36 & 4.64 \\
\hline Tabasco & 55748 & 57.89 & 32275 & 31708 & 567 & 5 & 0 & 0 & 0 & 5 & 1.57 & 0 \\
\hline Tamaulipas & 51694 & 105.90 & 54744 & 54464 & 280 & 27 & 0 & 0 & 0 & 27 & 4.95 & 0 \\
\hline Tlaxcala & 24142 & 102.51 & 24749 & 24565 & 184 & 15 & 1 & 1 & 0 & 13 & 5.29 & 0.40 \\
\hline Veracruz & 95155 & 87.83 & 83573 & 80609 & 2964 & 24 & 4 & 0 & 3 & 17 & 2.10 & 0.35 \\
\hline Yucatán & 21101 & 85.34 & 18008 & 17098 & 910 & 16 & 5 & 9 & 2 & 0 & - & 6.10 \\
\hline Zacatecas & 23581 & 133.92 & 31580 & 31405 & 175 & 23 & 8 & 2 & 0 & 13 & 4.13 & 0.63 \\
\hline TOTAL & 1361850 & 101.31 & 79717 & 54102 & 25615 & 882 & 173 & 94 & 57 & 558 & 4.12 & 1.09 \\
\hline
\end{tabular}

* Total de nacidos vivos atendidos en unidades de salud de la SSA

₹ Para interpretar adecuadamente la cobertura hay que considerar que el tamiz neonatal también se practica en la consulta externa de las unidades de $1^{\text {er }}$ y 20 nivel, en niños de población abierta

Fuente: SSA/D G El; A nuarios Estadísticos, 1989-1998, SSA/SISPA: 1999-2002

1.09 x 10 000, pudiese ser una aproximación cercana al fenómeno real.

México es un país grande, con enormes contrastes y diferencias tanto geográficas como socioeconómicas que hacen de cada una de sus entidades federativas una región que debe analizarse por separado y en función de sus propias variables; sin embargo, en el caso del HTC las variaciones observadas en la cobertura y en la prevalencia probablemente dependan de tres factores, el primero de tipo operativo, esto es, falla en el proceso del tamiz desde la toma de la muestra hasta la confirmación del caso, lo cual influiría negativamente en la detección de enfermos y podría explicar las bajas prevalencias observadas en algunos 


\section{Cuadro V \\ EdAD PROMEDIO DE LOS CASOS DE HIPOTIRIODISMO CONGÉNITO, AL INICIO DEL TRATAMIENTO. MÉxico, 2001-2002} Estados promedio Edad promedio Días por arriba de la
en días, 2001 en días,2002 meta ideal (30 días)

\begin{tabular}{llll} 
N uevo León & 24.5 & 24.0 & -6.0 \\
\hline Distrito Federal & 50.5 & 32.5 & 2.5 \\
\hline Hidalgo & 42.5 & 36.5 & 6.5 \\
\hline Zacatecas & 44.33 & 37 & 7 \\
\hline Jalisco & 56.87 & 38.56 & 8.56 \\
\hline Tamaulipas & 42.4 & 39.55 & 9.55 \\
\hline N ayarit & 51.42 & 40.5 & 10.5 \\
\hline Aguascalientes & 43 & 42.5 & 12.5 \\
\hline Michoacán & 62.12 & 43.15 & 13.15 \\
\hline Sinaloa & 44 & 50 & 20 \\
\hline Chihuahua & 60 & 50 & 20 \\
\hline San Luis Potosí & 78.33 & 51.1 & 21.1 \\
\hline Baja California Sur & 66.5 & 53 & 23 \\
\hline Tlaxcala & 61.12 & 55.75 & 25.75 \\
\hline Q uerétaro & 49.66 & 55.91 & 25.91 \\
\hline Durango & 54 & 56 & 26 \\
\hline O axaca & 83.5 & 57.33 & 27 \\
\hline México & 60.89 & 61.17 & 31.17 \\
\hline Puebla & 70.14 & 67.91 & 37.91 \\
\hline Coahuila & SD & 68 & 38 \\
\hline Campeche & 71.25 & 70.25 & 40.25 \\
\hline Chiapas & 64 & 70.87 & 40.87 \\
\hline Guanajuato & 68.33 & 74.18 & 44.18 \\
\hline Baja California & 60.25 & 74.8 & 44.8 \\
\hline Colima & 61 & 76 & 46 \\
\hline Morelos & 106.16 & 80.14 & 50.14 \\
\hline Q uintana Roo & 62.66 & 81.16 & 51.16 \\
\hline Guerrero & 96.1 & 85 & 55 \\
\hline Tabasco & 55.75 & $\mathrm{SD}$ & $\mathrm{SD}$ \\
\hline Veracruz & 57.11 & $\mathrm{SD}$ & $\mathrm{SD}$ \\
\hline Sonora & 59.5 & $\mathrm{SD}$ & $\mathrm{SD}$ \\
\hline Yucatán & $\mathrm{SD}$ & $\mathrm{SD}$ \\
\hline & 60.26 & 56.19 & 25.09 \\
\hline
\end{tabular}

estados, como en el caso de Yucatán y Sinaloa; el segundo factor sería el genotipo poblacional, esto es, que la población de alguna región específica del país tuviese susceptibilidad genética hacia el hipotiroidismo, y por último, el factor ambiental, especialmente la deficiencia de yodo (leve, moderada o grave), de la que se tienen algunas evidencias. ${ }^{31}$
Una aportación importante de este estudio consiste en dar a conocer la tasa de prevalencia en cada uno de los estados de la República (cuadro IV), y en proponer el uso del concepto de "tasa de falla", que refleja el número teórico de casos perdidos y que idealmente debe ser cero. El análisis de ambas tasas puede ser un parámetro útil para conocer la eficiencia operativa de los programas de tamiz, especialmente en lo concerniente a sus últimos eslabones: la confirmación de los casos sospechosos y su registro en el sistema informático correspondiente.

Por otro lado, si bien en otras publicaciones se ha dado a conocer la prevalencia de cada uno de los tipos de HTC según su etiología, ${ }^{8}$ en esta ocasión se desglosa de acuerdo con el sexo, encontrándose así que tanto la ectopia como la agenesia tiroidea ocurren predominantemente en el sexo femenino, a diferencia del bocio y la dishormonogénesis, que no parecen tener esta tendencia. La predominancia del sexo femenino, a pesar de que se confirma en nuestro estudio, permanece sin explicación; sin embargo, se sabe que este fenómeno también ocurre en otros defectos al nacimiento como los de tubo neural y algunas malformaciones cardiacas. . $^{32,33}$

En relación con el alto número de pacientes sospechosos de HTC que fallecieron antes de la confirmación, podemos decir que hay estudios que demuestran un alto número de complicaciones en los neonatos con esta enfermedad. ${ }^{34} \mathrm{El}$ mecanismo que pudiese explicar por qué los RN afectados con HTC tienen mayor riesgo de complicaciones neonatales es que las hormonas tiroideas fetales son necesarias para la maduración final de varios órganos, especialmente para los pulmones. ${ }^{35,36}$ Ridaura y colaboradore ${ }^{37}$ publicaron un estudio en el que concluyen que los niños con HTC se encuentran en riesgo de fallecer en forma inesperada. Ellos estudiaron las causas de muerte y las enfermedades concomitantes en necropsias de niños hipotiroideos, y señalan que en esta enfermedad existen trastornos orgánicos sistémicos que cuando se asocian con el daño neurológico pueden provocar la muerte, particularmente por trastorno de la función cardiopulmonar y por alteración del mecanismo de deglución. Los resultados de nuestro estudio concuerdan con los de autores anteriores, puesto que observamos que de 882 sospechosos de HTC 42 fallecieron, esto es, 23.8 x 1 000; si comparamos esta cifra con la mortalidad infantil (menores de un año) registrada en el Instituto Nacional de Estadística, Geografía e Informática, en México, en el año 2001 (12.68 x 1 000), encontramos que tener un resultado sospechoso de tamiz (esto es, una TSH elevada, cuantificada en papel filtro) teóri- 
camente eleva el riesgo de fallecer en 1.9 veces. De allí que cuando se notifique a un RN como sospechoso de HTC se debe proceder de manera verdaderamente urgente para su localización, confirmación e inicio de tratamiento inmediato, no sólo para proteger la integridad neurológica y prevenir discapacidad, sino también para disminuir el riesgo de muerte. Las causas más comunes del fallecimiento de los sospechosos de esta muestra fueron la asfixia neonatal severa y la falla orgánica múltiple, y llama la atención que también en los sospechosos fallecidos predominan dos mujeres por un hombre, fenómeno contrario a lo habitualmente observado en la mortalidad de menores de un año, en la que suele predominar el sexo masculino.

La proporción de muestras que no cumplieron con los requisitos para su procesamiento fue baja; sin embargo, nos indica que el personal encargado de obtener la sangre necesita actualizar sus conocimientos mediante un programa de capacitación continua.

Los principales obstáculos para la localización y confirmación de los casos sospechosos fueron el domicilio falso y, en menor proporción, la renuencia de los padres a la realización de los estudios confirmatorios. Ambos factores reflejan el desconocimiento tanto de la enfermedad como de la utilidad del TN para la prevención de discapacidades en la infancia, y señalan que hay que sensibilizar a toda la población sobre la importancia de este tipo de pruebas.

En lo referente a las variables de edad de la madre, edad de gestación y peso del RN no encontramos, en esta muestra, ninguna que tenga asociación aparente con la enfermedad. Por otro lado, el comportamiento que muestran los datos clínicos es similar al referido por la literatura especializada sobre HTC.

A pesar de la eficacia general de los programas de TN para HTC los falsos negativos ocasionales pueden ocurrir, por ello es esencial que todos los prestadores de servicios de salud estén alertas y conozcan los datos clínicos que afectan a estos pacientes. De acuerdo con nuestros resultados, podemos sugerir que todos aquellos pacientes con hernia umbilical, ictericia, piel seca, estreñimiento y facies tosca deben ser considerados como casos sospechosos de HTC, independientemente del resultado del TN, y deben ser referidos a las unidades de salud correspondientes.

En conclusión, podemos decir que el programa de TN de la SSA, a pesar del trabajo hasta ahora realizado, presenta contrastes muy importantes tanto en cobertura como en las tasas de prevalencia y falla. Además, la mayoría de los estados rebasan el tiempo ideal para el inicio del tratamiento; notable excepción es el estado de Nuevo León, que realiza el proceso integral de TN en 24 días.
El conocimiento de esta información es útil para diseñar estrategias específicas para cada estado, y establecer prioridades para una mejor operación del TN en nuestro país.

\section{Agradecimientos}

Agradecemos a la enfermera especializada Blanca E. Aguirre Vélez su invaluable colaboración en la capacitación para la toma correcta de las muestras de tamiz, así como en la coordinación de la confirmación de los casos. Igualmente reconocemos el apoyo a la señora Violeta Hita Zamudio, en el proceso de notificación de sospechosos.

\section{Referencias}

1. D eG root LJ, Larsen PR, Hennemann G. The thyroid and its diseases. $6^{\text {th }}$ ed. N ew York (N Y): C hurchill Livingston; 1996:33-112.

2. D ussault JH. The anecdotal history of screening for congenital hypothyroidism. J Clin Endocrinol Metab 1999;84:4332-4334.

3. LaFranchi S, D ussault JH, Fisher DA, Foley TP, Mitchell ML. N ewborn screening for congenital hypothyroidism: Recommended guidelines. Pediatrics 1993;91:1203-1209.

4. Secretaría de Salud. N ormaTécnica 321, para la Prevención del Retraso Mental Producido por Hipotiroidismo Congénito. México, DF: Diario 0 ficial de la Federación. 22 de septiembre de 1988;Tomo CDXX N o. 14:88-90.

5. Secretaría de Salud. N orma 0 ficial Mexicana-007-SSA2-1993, para la Atención a la Mujer Durante el Embarazo, Parto y Puerperio y del RN, Criterios y Procedimientos para la Prestación del Servicio. México, DF: Diario 0 ficial de la Federación; viernes 6 de enero de 1995;Tomo CDXCVI N 0. 5:19-38.

6.Velázquez A, Loera-Luna A, A guirre BE, Gamboa S,Vargas H, Robles C. Tamiz neonatal para hipotiroidismo congénito y fenilcetonuria. Salud Publica Mex 1994;36:249-256.

7. Loera-Luna A,A guirre BE, Gamboa S,Vargas H, Robles C, Velázquez A. Resultados del Programa para la Prevención del Retraso Mental Producido por Hipotiroidismo Congénito. Bol Med Hosp Infant Mex 1996;53:259-263.

8.Vela M, G amboa S, Loera-Luna A, A guirre B, Pérez-Palacios G, Velázquez $A$. N eonatal screening for congenital hypothyroidism in Mexico: Experience, obstacles, and strategies. J Med Screen 1999;6:7779.

9. Toublanc JE. Comparision of epidemiological data on congenital hypothyroidism in Europe with those of other parts in the world. Horm Res 1992;38:230-235.

10. Klett M. Epidemiology of congenital hypothyroidism. Exp C lin Endocrinol Diabetes 1997;105 Suppl 4:19-23.

11. Lorey FW, C unningham GC. Birth prevalence of primary hypothyroidism by sex and ethnicity. Hum Biol 1992;64:531-538. 12. Brown AL, Fernhoff PM, Milner BA, MCEwen, Elsas LS. Racial differences in the birth prevalence of congenital hypothyroidism. J Pediatr 1981;99:934-936.

13. Frasier SD, Penny R, Snyder R. Primary congenital hypothyroidism in Spanish-surnamed infants in Southern California. J Pediatr 1982;101:315. 
14. Penny R, Hoffamn P, Barton L. Congenital hypothyroidism in Spanishsurnamed infants in Southern California: Increased incidence and clustering of occurrence. Am J D is Child 1989;143:640-641.

15. W aller DK,Anderson JL, Lorey F, C unningham GC. Risk factor for congenital hypothyroidism:An investigation of infant's birth weight, and gender in California, 1990-1998. Teratology 2000;62:36-41.

16. Delange F, Heidemann P, Bourdoux P, Larsson A, Vigneri R, Klett $M$ et al. Regional variations of iodine nutrition and thyroid function during the neonatal period in Europe. Biol N eonate 1986;49:322-330.

17. D elange F. Screening for congenital hypothyroidism used as an indicator of the degree of iodine deficiency and of its control.Thyroid 1998;8:1185-1192.

18. Charoensiriwatana W, Janejai N , Boonwanich W, Tananchai P. Monitoring of the severity of iodine deficiency areas in Thailand using neonatal blood spots. $4^{\text {th }}$ Meeting of the International Society for N eonatal Screening; 1999 June 13-16. Stockholm, Sweden:76.

19. D evos H, Rodd C, Gagné N , Laframboise R,Van Vliet G.A search for the possible molecular mechanisms of thyroid dysgenesis: Sex ratios and associated malformations. J C lin Endocrinol Metab 1999;84:2502-2506. 20. Gillam MP, C op P. Genetic regulation of thyroid development. Curr 0 pinion Pediatr 2001;13:358-363.

21. Mansouri A, Chowdhury K, Gruss P. Follicular cells of the thyroid gland requires Pax8 gene function. N at G enet 1998;19:87-90.

22. De Felice $M, O$ vita $C$, Biffali E.A mouse model for hereditary thyroid dysgenesis and cleft palate. $N$ at Genet 1998;19:395-326.

23. Abramowicz MJ,Vassart G, Refetoff S. Probing the cause of thyroid dysgenesis. Thyroid 1997;7:325-326.

24. Krude H, Macchia PE, Di Lauro R, Grüters A. Familial hypothyroidism due to thyroid dysgenesis caused by dominant mutations of the PAX 8 gene. Horm Res 1998;50 Suppl 3:17.

25. Biebermann $\mathrm{H}$, Liesenkötter KP, Emeis $\mathrm{M}, \mathrm{O}$ bladen $\mathrm{M}, \mathrm{G}$ rüters $\mathrm{A}$. Severe congenital hypothyroidism due to a homozygous mutation of the TSH. Gene Pediatr Res 1999;46:170-173.

26. Clifton-Bligh RJ,W entworth JM, H einz P, C risp MS, John R, Lazarus $J H$ et al. Mutation of the gene encoding human TTF-2 associated with thyroid agenesis, cleft palate and choanal atresia. N at Genet 1998;19: 399-401.

27. Kremer RD. Filter paper in clinical diagnostic screening. C lin Lab Prod 1982;10:21-25.

28. Therrell BL, Panny SR, D avidson A, Eckman J, Hannon W H, Henson MA et al. US N ewborn screening system guidelines: Statement of the Council of Regional N etworks for Genetic Services. Screening 1992;1: 135-147.

29. Centers for Disease Control and Prevention-Association of Public Health Laboratories. N ewborn Screening Q uality Assurance Program. Atlanta (GA): CDC-APHL; 2003.

30. Elandt-Johnson RC. D efinition of rates: Some remarks on their use and misuse.Am J Epidemiol 1975;102:267-271.

31.Vela-A mieva M, Hernández-O sorio C, G amboa-C ardiel S, G onzálezContreras CR, Pérez-Andrade ME, 0 rtiz-Cortés J et al. Hipertirotropinemia en recién nacidos mexicanos. Salud Publica Mex 2003:45:269-275.

32. Seller MJ. N eural tube defects and sex ratios. Am J Med Genet 1987; 26:699-707.

33. Samanek M. Boy:G irl ratio in children born with different forms of cardiac malformation: A population-based study. Pediatr Cardiol 1994; 15:53-57.

34. Fernhoff PM, Brown AL, Elsas LJ. Congenital hypothyroidism: Increased risk of neonatal morbidity results in delayed treatment. Lancet 1987;1(8531):490-491.

35. Fisher A, Klein AH. Thyroid development and disorders of thyroid function in the newborn. N Engl J Med 1981;304:702-712.

36. Erenberg A, Rodees ML,W einstein MM, Kennedy RL.The effect of fetal thyroidectomy on ovine fetal lung maturation. Pediatr Res 1979;13: 230-235.

37. Ridaura-Sanz C, López-Corella E. Las causas de muerte por hipotiroidismo congénito. Un estudio realizado en necropsias. $\mathrm{Gac}$ Med Mex 1995;131:141-146. 